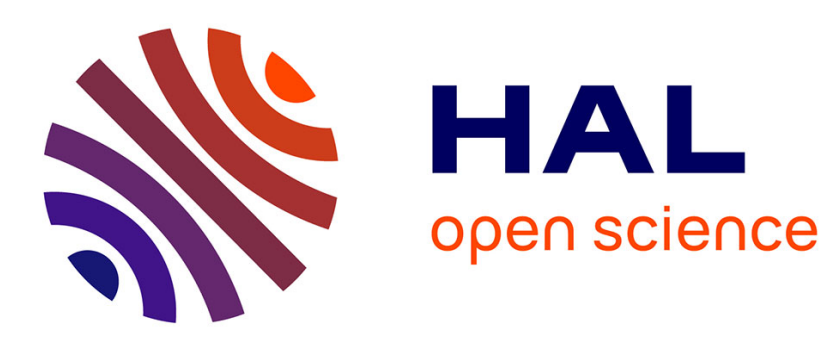

\title{
Dispositif de compensation automatique du vecteur champ magnétique terrestre
}

\author{
G. Argence
}

\section{To cite this version:}

G. Argence. Dispositif de compensation automatique du vecteur champ magnétique terrestre. Revue de Physique Appliquée, 1970, 5 (1), pp.159-163. 10.1051/rphysap:0197000501015900 jpa-00243352

\section{HAL Id: jpa-00243352 https://hal.science/jpa-00243352}

Submitted on 1 Jan 1970

HAL is a multi-disciplinary open access archive for the deposit and dissemination of scientific research documents, whether they are published or not. The documents may come from teaching and research institutions in France or abroad, or from public or private research centers.
L'archive ouverte pluridisciplinaire HAL, est destinée au dépôt et à la diffusion de documents scientifiques de niveau recherche, publiés ou non, émanant des établissements d'enseignement et de recherche français ou étrangers, des laboratoires publics ou privés. 


\title{
DISPOSITIF DE GOMPENSATION AUTOMATIQUE DU VEGTEUR GHAMP MAGNÉTIQUE TERRESTRE
}

\author{
Par G. ARGENGE, \\ Laboratoire Pierre-Weiss, 3, rue de l'Université, 67-Strasbourg (France).
}

\begin{abstract}
Résumé. - On discute les types de dispositifs d'annulation du vecteur champ magnétique terrestre par bobines de compensation. On étudie, en particulier, les conditions optimales de compensation, en fonction de divers types de sondes de champ, d'un système original " matriciel " de bobines de "reproduction"; on montre que cet agencement le plus général de bobines est optimal.

On indique les performances d'un dispositif de compensation automatique du vecteur champ magnétique terrestre (utilisant des sondes horizontales du type variomètre à quartz, à faible champ directeur stabilisé automatiquement) réalisé sur les bases de cette étude.
\end{abstract}

Introduction. - Les domaines de la Physique nécessitant l'annulation locale d'un champ magnétique (ou, plus généralement, l'obtention d'un champ quasi nul, finement contrôlable en module et direction) sont de plus en plus nombreux, comme, depuis peu, la simulation magnétique spatiale.

Dans le cadre de ce colloque, toutes les méthodes d'obtention d'un champ nul décrites concernent des blindages soit supraconducteurs, soit ferromagnétiques [1]. Cet exposé traite des méthodes d'annulation, plus classiques, par bobines de compensation et indique, en particulier, un agencement optimal de ces bobines associé à un type de détecteurs de champ optimal.

Les « blindages », comparés aux « bobines de compensation ", permettent d'atteindre des champs résiduels plus faibles [par exemple $\sim 10^{-8} \mathrm{~g}$ [1] contre $\simeq 3 \times 10^{-7} \mathrm{~g}$ (cet article)]. Toutefois, ces dernières ont à leur avantage (à l'heure actuelle) un accès plus aisé, une finesse de contrôle supérieure; surtout, elles seules sont utilisables dans le cas où l'ensemble physique, qui doit être placé en champ nul, produit luimême un champ magnétique ou électrique (non négligeable).

Méthodes d'annulation par bobines de compensation. - Le principe général, classique, consiste à réguler les 3 courants indépendants de 3 ensembles de bobines (à homogénéité de champ maximale [2]) définissant 3 axes magnétiques, au moyen de 3 détecteurs de composantes de champ.

a) Systèmes SANS « BOBINES DE REPRODUGTION ». Une première solution, simple, mais d'application très restreinte, consiste à placer les 3 détecteurs au voisinage immédiat du centre $\mathrm{O}$ commun du système de bobines, où le champ doit être maintenu nul [3]. Les conditions d'utilisation sont alors draconiennes :

1) Il faut éviter les interactions magnétiques entre détecteurs : pratiquement, seules sont utilisables les sondes «fluxgate », de stabilité et résolution assez moyennes.

2) Il faut que le voisinage du point $\mathrm{O}$ soit libred'accès, ce qui n'est en général pas le cas, puisque s'y trouve placé l'ensemble physique testé, souvent volumineux.
3) Il faut que cet ensemble physique soit neutre électriquement et magnétiquement, de façon à ne pas perturber les 3 détecteurs.

4) Il faut une corrélation vectorielle parfaite du champ des bobines en $\mathrm{O}$ et au niveau des 3 détecteurs, ce qui implique des dimensions de bobines considérables.

b) Systèmes aveG bobines de Reprodugtion. On placera donc, en général, les 3 sondes à une distance suffisante du centre $\mathrm{O}$, de façon que les interactions entre le système d'annulation principal et l'ensemble physique placé en $\mathrm{O}$, d'une part, et chacun des détecteurs de champ et de leurs bobines associées, d'autre part, puissent être négligées (sinon corrigées linéairement), mais minimale de façon à réduire l'influence du gradient du champ local.

Dans ce cas, on peut envisager plusieurs possibilités :

- Aucune bobine n'est associée aux détecteurs (on ne compense dans ce cas que les fluctuations autour d'une valeur moyenne [4]. Il y a alors deux contraintes sérieuses :

a) Il faut un détecteur de champ très linéaire, d'étalonnage très stable (seule est alors acceptable une sonde type nucléaire ou à pompage optique);

$\beta$ ) Il faut une extrême stabilité en courant (pour la compensation de la valeur moyenne).

- On utilise une méthode de contre-réaction : on est alors amené à réaliser des « reproductions », plus ou moins complètes du système de bobines principales, centrées sur les 3 détecteurs de champ respectifs. Dans la suite, nous discutons d'emblée le cas le plus général d'une reproduction « complète ».

Description de principe du système " matriciel " (complet) de bobines et de ses détecteurs de champ associés. - Il comprend :

a) UN SYSTÈme DE BOBINES « PRINGIPAL », 3 ensembles de bobines, d'axes magnétiques $L, M, N$ approximativement orthogonaux :

$$
\left(\begin{array}{l}
\mathbf{H}_{L}=\mathbf{K}_{L} i_{L} \\
\mathbf{H}_{M}=\mathbf{K}_{M} i_{M} \\
\mathbf{H}_{N}=\mathbf{K}_{N} i_{N}
\end{array}\right)
$$


représentent les champs magnétiques associés aux 3 ensembles $L, M, N \quad(|\mathbf{K}|=$ constante de champ; $i=$ courant).

b) Trois Systèmes DE BOBINES DE REPRODUGTION $(l, m, n)$, affectés chacun à la compensation d'une des 3 composantes du champ.

Chacun de ces systèmes consiste en une réplique (miniaturisée) aussi fidèle que possible du système principal; donc, chacun de ces systèmes comprend 3 paires de bobines (d'axes $l, m, n$ ). Au système principal se trouve ainsi associé le système de reproduction « matriciel » suivant :

$$
\left(\begin{array}{l}
\mathbf{h}_{l} \\
\mathbf{h}_{m} \\
\mathbf{h}_{n}
\end{array}\right)=\left(\begin{array}{lll}
\mathbf{k}_{l, l} & \mathbf{k}_{l, m} & \mathbf{k}_{l, n} \\
\mathbf{k}_{m, l} & \mathbf{k}_{m, m} & \mathbf{k}_{m, n} \\
\mathbf{k}_{n, l} & \mathbf{k}_{n, m} & \mathbf{k}_{n, n}
\end{array}\right)\left(\begin{array}{l}
i_{L} \\
i_{M} \\
i_{N}
\end{array}\right)
$$

(branchement série des bobines d'axes parallèles à une même composante).

$\mathbf{h}_{l}=\mathbf{k}_{l, l} i_{L}+\mathbf{k}_{l, m} i_{M}+\mathbf{k}_{l, n} i_{N} \quad$ représente, par exemple, le champ au centre du système de reproduction affecté à la compensation de la composante $\mathbf{L}$.

c) Trois détecteurs De GHAMP, placés aux centres des 3 systèmes de reproduction. On peut les classer en sondes (1) :

- Directives. - Type $\alpha$ ) : vectorielles («fluxgate », « Hall », etc.).

Type $\beta$ ) : « pseudo-vectorielles » (balances magnétiques, variomètres à quartz...).

On peut les considérer, en grossière approximation, comme des vecteurs $\left(\mathbf{P}_{l}, \mathbf{P}_{m}, \mathbf{P}_{n}\right)$.

- Scalaires. - Type $\gamma$ ) (résonance nucléaire, pompage optique, etc.) dont on néglige la faible anisotropie.
Les sondes type $\gamma$ ) pour cette compensation vectorielle et certaines du type $\beta$ ) nécessitent des champs directeurs, notés $\left(\mathbf{D}_{l}, \mathbf{D}_{m}, \mathbf{D}_{n}\right)$. Dans le cas présent, ce sont les champs effectifs aux centres des 3 systèmes de reproduction : ils sont la somme de champs «supplémentaires » $\left(\mathbf{D}_{l}^{\prime}, \mathbf{D}_{m}^{\prime}, \mathbf{D}_{n}^{\prime}\right)$ intentionnellement appliqués [il est alors commode d'utiliser un bobinage supplémentaire sur les bobines $\mathbf{k}_{i, i}(\forall i=l, m, n)$ (sondes $\gamma$ ) ou $\mathbf{k}_{i, j}(\forall i \neq j)$ (sondes $\beta$ ), déjà existantes] et des champs résiduels (après compensation au centre du système principal) aux centres des 3 systèmes de reproduction.

Conditions de compensation optimale (par reproduction). - La compensation serait parfaite (quel que soit le type de sondes), si les conditions suivantes, au moins, étaient remplies :

1) $\left(\mathbf{K}_{L}, \mathbf{K}_{M}, \mathbf{K}_{N}\right)=$ base vectorielle strictement orthogonale;

2) a) Reproduction parfaite (en module et suivant les 3 directions) du système de bobines principal;

b) Corrélation vectorielle parfaite du champ local aux centres des 4 systèmes ( $a$ étant, sinon, ajustée en fonction de $b$ );

3) Champs directeurs (si nécessaires), strictement parallèles aux axes des bobines, et stabilisés en module;

4) Gain cyclique infini ou, sinon, courants primaires parfaitement stabilisés (suivant que les bobines de compensation sont à un ou deux bobinages, le deuxième étant alors destiné à la compensation des fluctuations seules).

En fait, on peut effectuer un classement en conditions au premier et au deuxième ordre; on s'attache donc à satisfaire au mieux aux premières.

Fonctionnement approché du dispositif de compensation. - Notations (angulaires) :

$$
\begin{aligned}
& \text { SONDES DIREGTIVES } \\
& \left.\begin{array}{l}
\eta_{i, j}=\left(\mathbf{P}_{i}, \mathbf{K}_{j}\right) \\
\varepsilon_{i, j}=\left(\mathbf{P}_{i}, \mathbf{k}_{i, j}\right)
\end{array}\right\}(\forall i, j=l, m, n) ; \quad\left\{\begin{array}{l}
\eta_{i, j}=\left(\mathbf{D}_{i}, \mathbf{K}_{j}\right) \\
\varepsilon_{i, j}=\left(\mathbf{D}_{i}, \mathbf{k}_{i, j}\right)
\end{array}\right. \\
& \beta_{i, j}=\left(\mathbf{k}_{i, j}, \mathbf{K}_{j}\right) .
\end{aligned}
$$

Une variation $\Delta \mathbf{H}$ du champ local (supposé en outre uniforme) est décomposable univoquement suivant les axes du système principal : $\Delta \mathbf{H}=\Delta \mathbf{L}+\Delta \mathbf{M}+\Delta \mathbf{N}$. La compensation est " parfaite " si, et seulement si, une variation $\Delta \mathbf{J}(\forall J=L, M, N)$ induit :

- une composante de champ $\mathbf{K}_{J} \Delta i_{J}$ égale et opposée, - aucune composante « parasite »: $\mathbf{K}_{I} \Delta i_{I}=0(\forall I \neq J)$.

Sondes type $\alpha)$. - Dans ce cas, on a les conditions approchées :

$$
\left\{\begin{array}{l}
\varepsilon_{i, i}, \eta_{i, i} \simeq 0(\forall i=l, m, n) \\
\varepsilon_{i, j}, \eta_{i, j} \simeq \frac{\pi}{2}(\forall i \neq j) .
\end{array}\right.
$$

Une variation $\Delta \mathbf{L}$, par exemple, du champ local, induira des variations de courants $\Delta i_{L}, \Delta i_{M}, \Delta i_{N}$, solutions du système :
(1) On ne s'occupe ici que de sondes mesurant le continu. 
$\left\{\begin{array}{l}\mathbf{P}_{l} \cdot\left(\mathbf{k}_{l, l} \Delta i_{L}+\mathbf{k}_{l, m} \Delta i_{M}+\mathbf{k}_{l, n} \Delta i_{N}\right)+\mathbf{P}_{l} \cdot \Delta \mathbf{L}=0 \\ \mathbf{P}_{m} \cdot\left(\mathbf{k}_{m, l} \Delta i_{L}+\mathbf{k}_{m, m} \Delta i_{M}+\mathbf{k}_{m, n} \Delta i_{N}\right)+\mathbf{P}_{m} \cdot \Delta \mathbf{L}=0 \\ \mathbf{P}_{n} \cdot\left(\mathbf{k}_{n, l} \Delta i_{L}+\mathbf{k}_{n, m} \Delta i_{M}+\mathbf{k}_{n, n} \Delta i_{N}\right)+\mathbf{P}_{n} \cdot \Delta \mathbf{L}=0 .\end{array}\right.$

D'où les variations de composantes de champ induites au centre du système principal (négligeant des termes d'ordre supérieur) :

$$
\begin{aligned}
-\Delta H_{L} \simeq \frac{K_{L}}{K_{l, l}} \Delta L & {\left[l+\frac{\varepsilon_{l, l}^{2}}{2}-\frac{\eta_{l, l}^{2}}{2}-\varepsilon_{l, m} a_{m, l}-\varepsilon_{l, n} a_{n, l}\right] } \\
& -\Delta H_{M} \simeq a_{m, l} \Delta L
\end{aligned}
$$$$
\text { où } a_{m, l}=\left(\eta_{m, l}-\frac{k_{m, l}}{k_{l, l}} \varepsilon_{m, l}\right)
$$$$
-\Delta H_{N} \simeq a_{n, l} \Delta L
$$

(de même $a_{n, l}$ ).

Sondes type $\beta$ ). - Dans ce cas, il n'y a pas isotropie pour les 3 directions $(l, m, n)$. L'une joue un rôle particulier (verticale : variomètres à quartz, horizontale : balance magnétique verticale). Par construction, une variation du champ suivant cette direction privilégiée ( $n$ par exemple) n'aura qu'un faible effet (mais non complètement négligeable en général) sur la sonde. Si l'on suppose cet effet simplement nul au deuxième ordre près (ce qui est pratiquement toujours le cas), et avec les hypothèses :

$$
\begin{cases}\varepsilon_{i, j}, \eta_{i, j} \simeq 0 & (\forall i, j=l, m ; i \neq j) \\ \varepsilon_{i, i}, \eta_{i, i} \simeq \frac{\pi}{2} & (\forall i=l, m)\end{cases}
$$

on trouve alors des expressions de $\Delta H_{L}, \Delta H_{M}$, tout à fait analogues à celles obtenues pour les sondes type $\alpha$ ).
Sondes type $\gamma$ ) (mêmes hypothèses que sondes type $\alpha$ ) : Écrivant $\quad\left|\mathbf{D}_{j}\right|=$ constante $\quad(\forall j=l, m, n)$, on trouve :

$$
\begin{aligned}
& \Delta H_{L} \simeq-\frac{K_{L}}{k_{l, l}} \Delta L\left[1+\frac{\varepsilon_{l, l}^{2}}{2}-\frac{\eta_{l, l}^{2}}{2}\right. \\
& \left.+\frac{\Delta L}{D_{l}} \frac{\beta_{l, l}^{2}}{2}-\varepsilon_{l, m} a_{m, l}-\varepsilon_{l, n} a_{n, l}\right] . \\
& \Delta H_{M} \simeq-\Delta L\left[a_{m, l}+\frac{\Delta L}{2 D_{m}} b_{m, l}\right] \\
& \text { où } b_{m, l}=\left(1-\frac{k_{m, l}}{k_{l, l}}\right)^{2}+\beta_{m, l}^{2} \\
& \Delta H_{N} \simeq-\Delta L\left[a_{n, l}+\frac{\Delta L}{2 D_{n}} b_{n, l}\right]
\end{aligned}
$$

(de même $b_{n, l}$ ).

Donc ce type de sondes semble à peine moins favorable que les types $\alpha$ ) et $\beta$ ).

Dans les systèmes de compensation (par « reproduction ») [5], ou de mesure de composantes de champ (par compensation) [6], réalisés jusqu'ici par d'autres auteurs, les termes non diagonaux de (1) (cas des sondes directives) sont, à notre connaissance, omis (c.-à-d. $k_{i, j}=0, \forall i \neq j$ ). Dans le cas de sondes scalaires, certaines des bobines de type $k_{i, j}(i \neq j)$ existent forcément (afin de rendre la sonde scalaire " directive »), mais leurs courants ne sont en général pas régulés [7] $\left(k_{i, j} \Delta i_{j}=0 \forall i \neq j\right)$.

Aussi indiquons-nous, à titre comparatif, les conditions (au premier ordre) telles qu'elles résultent de l'étude précédente :
SystÈme « MATRIGIEL » (complet)

1) Conditions «d'égalisations »: $\left|\mathbf{K}_{i}\right|=\left|\mathbf{k}_{i, i}\right| \forall i=l, m, n$

2) Conditions «d'alignement»: $\left(\mathbf{K}_{i}, \mathbf{K}_{j}\right)=\left(\mathbf{K}_{i}, \mathbf{k}_{i, j}\right) \forall i \neq j$

\section{SYSTÈmE « DIAGONAL 》}

$$
\begin{gathered}
\text { idem } \\
\begin{cases}\text { sondes } \alpha) & : \mathbf{P}_{i} \cdot \mathbf{K}_{j}=0 \\
\text { sondes } \beta \text { ) } & : \mathbf{P}_{i} \Lambda \mathbf{K}_{j}=0 \\
\text { sondes } \gamma \text { ) } & : \mathbf{D}_{i} \Lambda \mathbf{K}_{j}=0\end{cases}
\end{gathered}
$$

plus la condition, pour les deux systèmes, $\left|\mathbf{D}_{i}^{\prime}\right|=$ Cte (sondes $\gamma$ ). Les avantages du système matriciel par rapport au système diagonal, en contrepartie de sa plus grande complexité, découlent des comparaisons des conditions 2 ).

$\alpha$ Les champs associés aux bobines $k_{i, j}(i \neq j)$ ont toutes les propriétés d'un « vrai » vecteur, ce qui n'est pas le cas des sondes directives $\mathbf{P}_{i}$ (propriétés tensorielles, non-linéarité, etc.). Elles ont, de plus, un gradient beaucoup plus important; en conséquence, la justesse, la précision et même la stabilité directionnelle des bobines $k_{i, j}$ nous paraissent meilleures que celles des sondes $\mathbf{P}_{i}$.

$\beta)$ Il est pratiquement très difficile d'aligner une sonde perpendiculairement à la fois à deux directions (condition $2 \alpha$ ) ou parallèlement à une direction donnée, tout en la rendant strictement insensible à une autre composante $(2 \beta)$. Ceci est remplacé par un alignement précis de deux bobines.

$\gamma)$ Le système matriciel, une fois en place, permet le remplacement immédiat de la sonde par n'importe quelle autre sonde, même d'un type différent. Dans le cas d'une sonde "directive », il suffit de l'aligner " au deuxième ordre près " (par exemple, à $\sim 10$ à $2^{o}$ près, pour une compensation à $10^{-3}$ près, au lieu de $3^{\prime}$ près).

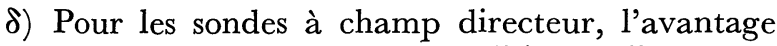
est notable : étant donné cette condition d'alignement au deuxième ordre seulement, on peut descendre à un champ directeur beaucoup plus faible, sans perdre en « justesse »; on verra que, dans le cas des sondes scalaires, on gagne en stabilité, mais surtout, dans le cas de sondes (horizontales) type aimant suspendu (à torsion quasi nulle), on gagne et en sensibilité et en stabilité.

Cas des sondes à champ directeur. - D'après ce qui précède, l'orientation des sondes (directives) ou des champs directeurs (sondes scalaires) est une condition du deuxième ordre ( $\mathrm{du}$ premier pour tout système diagonal). Or cet alignement est fonction, d'une part de l'alignement du champ appliqué $\mathbf{D}_{j}^{\prime}$ (qu'on peut 
considérer comme réalisé au deuxième ordre), et surtout de celui, a priori quelconque, du faible champ résiduel $\Delta h_{j}$ au centre du système de reproduction $j$ $(j=l, m, n)$.

Au départ, les courants $i_{L}, i_{M}, i_{N}$ sont ajustés de façon que le champ résiduel au centre du système principal soit nul.

$$
\begin{aligned}
& \text { Sondes type } \gamma) \text {. - Hypothèses : } \\
& \qquad\left\{\begin{array}{l}
\mathbf{D}_{j}^{\prime} \Lambda \mathbf{K}_{j}=0 \\
\Delta h_{j} \ll \mathbf{D}_{j}^{\prime} .
\end{array}\right.
\end{aligned}
$$

On trouve :

$$
\begin{aligned}
D_{l}^{\prime}\left|\eta_{l, m}\right| \leqslant\left|\frac{\left[K_{M}-k_{l, m}\right] M}{K_{M}}\right| & \\
& +\left|\left[\left(\mathbf{K}_{L}, \mathbf{K}_{M}\right)-\left(\mathbf{k}_{l, l}, \mathbf{K}_{M}\right)\right] L\right| \\
& +\left|\left[\left(\mathbf{K}_{N}, \mathbf{K}_{M}\right)-\left(\mathbf{k}_{l, n}, \mathbf{K}_{M}\right)\right] N\right|
\end{aligned}
$$

(+ permutation sur $l, m, n) ; L, M, N$ désignent les composantes du champ local.

On constate que les quantités entre crochets ne figurent pas dans les conditions « au premier ordre » indiquées plus haut.

Il en résulte que, à moins :

- ou bien de réaliser des égalisations et des alignements de toutes les bobines $k_{i, j}$ au premier ordre près;

- ou bien d'introduire des bobinages correctifs (après mesure des composantes résiduelles aux centres des systèmes de reproduction);

il n'est pas possible, sans perdre en justesse, de descendre à un champ directeur inférieur en ordre de grandeur, au champ terrestre (ce qui nécessiterait alors une stabilisation des courants produisant $\mathbf{D}_{j}^{\prime}$, exceptionnelle, de l'ordre de $\simeq 2 \times 10^{-6}$ pour une compensation à $\simeq 0,1 \gamma$ près, par exemple).

Sondes types variomètres à quartz. - Supposant (pour alléger) l'effet d'un champ vertical négligeable, et tous les vecteurs coplanaires, on a (fig. 1) :

$$
\left|\eta_{l, m}\right|<\frac{\left|\frac{\left(K_{L}-k_{l, l}\right) L}{K_{L}}\right|+\left|\beta_{m, l} M\right|}{D_{L}^{\prime}+\left|\frac{\left(K_{M}-k_{l, m}\right) M}{K_{M}}\right|+\left|\beta_{l, l} L\right|}+\left|\frac{C \varphi_{0}}{P_{L} D_{L}^{\prime}}\right| .
$$

Dans ce cas, le numérateur du premier terme est nul au premier ordre. On peut donc facilement, ici, abaisser le champ directeur (par exemple avec une composante $N-S$ de $\simeq 2 \times 10^{4} \gamma$, conserver une « justesse » en compensation de $\simeq 10^{-3}$, avec $D_{L} \simeq 500 \gamma$ ).

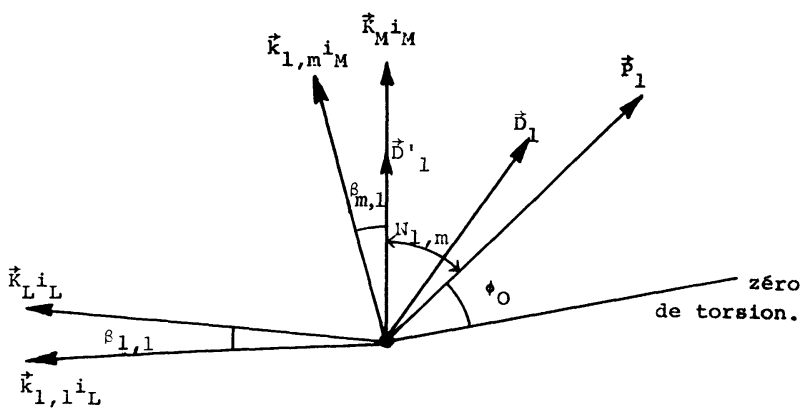

FIG. 1. - Variomètres horizontaux.

Erratum : Sur la figure au lieu de $\mathrm{N}_{\mathrm{l}, \mathrm{m}}$, lire $\eta_{l . m}$.
Stabilité du dispositif matriciel associé à une sonde type variomètre à quartz. - Dans ce cas particulier de sonde, la stabilité est liée à la sensibilité.

On a : $\sigma \simeq \frac{1}{D_{l}}$ (sensibilité statique) (fig. 2), [8] :

$\Delta L_{\text {équ }} \simeq \frac{\varphi_{0} C}{P_{l}}\left[ \pm \frac{\Delta P_{l}}{P_{l}} \pm \frac{\Delta D_{l}}{D_{l}} \pm \frac{\Delta C}{C}\right]+D_{l} \frac{[ \pm \Delta u \pm \Delta v]}{1 \pm C / P_{l} D_{l}}$

où $\Delta L_{\text {équ }}$ représente la variation équivalente, parasite, en champ, qui résulte des dérives $\pm \Delta$ du deuxième

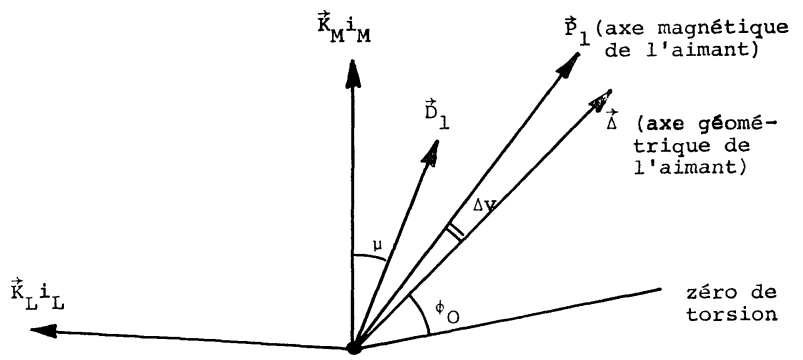

FIG. 2. - Compensation de la composante $\mathbf{L}$ :

$\mathbf{D}_{1}^{\prime}$, champ directeur appliqué ; $\mathbf{D}_{1}$, champ directeur total $(\geqslant 500 \gamma) ; \mathbf{P}_{1}$, moment magnétique de l'aimant; $C$, constante de torsion de la suspension de l'aimant; $\varphi_{0}$, azimut de torsion de l'aimant $\left(C / P_{L} \leqslant 2,5 \gamma\right.$; $\varphi_{0} \leqslant \pm 5^{\circ}$ ).

membre. On notera que la dérive de la sonde (avec les valeurs expérimentales) est pratiquement négligeable (quelle qu'en soit la cause), pour des champs directeurs suffisamment faibles.

Conclusions. - On a montré que dans le cadre d'un dispositif d'annulation du vecteur champ magnétique terrestre, par bobines de compensation, le système « matriciel » de bobines de reproduction est le plus précis et applicable à tout type de sondes.

Le type de sondes (dans le cadre de ce système) le mieux adapté (pour les composantes horizontales) consiste en de simples variomètres à quartz, à faible champ directeur (qui est alors stabilisé automatiquement en direction et en module).

Ces conclusions sont aussi applicables au cas des mesures de composantes de champ par contre-réaction. Il nous semble qu'un dispositif « matriciel » (si l'on mesure en un même lieu les 3 composantes) est plus juste (et plus cohérent) qu'un système « diagonal » (type « Dürschner ») [6].

Application. - Nous avons réalisé un dispositif «matriciel » de compensation automatique du vecteurchamp magnétique terrestre, d'après l'étude précédente, utilisant des sondes type aimant suspendu (à torsion quasi nulle) (composantes horizontales) et type «fluxgate » $\left({ }^{2}\right)$ (composante verticale).

Performances. - Compensation des fluctuations des 3 composantes à $\leqslant \pm 10^{-3}$ près; bruit, résolution $\leqslant \pm 0,03 \gamma$ (horizontal); champ directeur $\simeq 2000 \gamma$; stabilité $(48 \mathrm{~h}): \leqslant \pm 0,1 \gamma$ (horizontal) $; \leqslant \pm 0,25 \gamma$ (vertical).

(2) Sondes Förster; Reutlingen (R.F.A.). 


\section{BIBLIOGRAPHIE}

[1] COHEN (D.).

[2] Freedman (M. S.) et al., J. Appl. Phys., 1967, 38, 1856 (contient de nombreuses références d'ensembles de bobines à haute homogénéité).

[3] Par exemple en magnétisme des roches.

[4] Graham et Geiger, Can. J. Phys., 1961, 39, 1357.

[5] Nous ne citons ici, parmi les nombreuses publications sur la compensation vectorielle du champ terrestre, que les plus importantes (à notre connaissance) : RöSSIGER (M.), Die Naturwissensch., 1942, 50, 753. NaUmann, Zeitschr. für angew. Phys., 1949, 1, 260.
ScOTT (G. G.), Rev. of Scient. Instrum., 1957, 28, 270.

Neison, GebHardT et BotTum, Publicat. of the U.S. coast and geodetic survey (Fredericksburg Magnetic Laboratory).

[6] Dürschner (H.), Annales de Géophys., 1951, 7, 199 (dispositif en fait postérieur à ceux de Rössiger et Naumann).

[7] Hurwitz et Nelson, J. of Geoph. Res., 1960, 65, 1759.

[8] THELliER (E.), Annals of the I.G.Y., IV, 5, 255. 